Bulgarian Academy of Sciences. Space Research and Technology Institute.

Aerospace Research in Bulgaria. 32, 2020, Sofia

DOI: https://doi.org/10.3897/arb.v32.e09

\title{
LONG ENDURANCE ELECTRIC MULTIROTOR UNMANNED AERIAL VEHICLE
}

\begin{abstract}
Dimo Zafirov
Space Research and Technology Institute - Bulgarian Academy of Sciences

e-mail: zafirov@space.bas.bg

Key words: Multi-Rotor UAV, Long Endurance, LiPo, LiION

Abstract

The article presents an algorithm for development of a Long endurance electric multirotor unmanned aerial vehicle. Calculations for usage of different types of electric batteries have been made and dependencies of flight time for different weights of batteries have been obtained. Options for quadcopter and sixcopter have been considered.
\end{abstract}

\section{Notation}

$C_{D}$ - coefficient of drag force;

$C_{L}-$ coefficient of lift force;

$\boldsymbol{D}$ - drag force of the aircraft;

$\boldsymbol{E}_{\text {bat }}$ - energy of the batteries;

$\overline{\boldsymbol{E}_{\boldsymbol{b a t}}}-$ specific energy of the batteries;

$\boldsymbol{F}$ - thrust of the propulsions;

$\boldsymbol{g}$ - acceleration of gravity;

$\boldsymbol{K}$ - glade ratio;

$\boldsymbol{K}_{e}$ - glade ratio by maximum endurance;

$\boldsymbol{K}_{\boldsymbol{R}}$ - glade ratio by maximum distance;

$\boldsymbol{L}$ - lift force of the aircraft;

$\boldsymbol{m}_{\boldsymbol{0}}$ - take-off mass;

$\boldsymbol{m}_{\boldsymbol{p}}$ - mass of the payload;

$\boldsymbol{m}_{\boldsymbol{b a t}}$ - mass of the batteries;

$\boldsymbol{m}_{\text {empty }}$ - empty mass of the aircraft;

$\overline{\boldsymbol{m}_{\boldsymbol{b a t}}}-$ specific mass of the batteries; 
$\overline{\boldsymbol{m}_{\boldsymbol{p}}}$ - specific mass of the payload;

$\overline{\boldsymbol{m}_{\text {empty }}}$ - specific mass of the empty aircraft;

$\boldsymbol{P}$ - power;

$\boldsymbol{P}_{1}$ - power for 1 motor;

$\boldsymbol{R}$ - distance of the flight;

$\boldsymbol{t}$ - flight time;

$\boldsymbol{t}_{\boldsymbol{e}}$ - endurance time;

$V$ - air speed of the aircraft;

$V_{c}$ - cruise speed of the aircraft;

$\boldsymbol{W}_{\mathbf{0}}$ - take-off weight;

$\boldsymbol{\rho}$ - air density.

\section{State of the Art}

In the past years, sales of multi-rotor unmanned aerial vehicles (UAV) (copters) represent a major part (over 90\%) of total sales of such aircraft. This is due to UAV capability to perform take-off and landing on small non-equipped areas, to carry out motionless hovering, and to be easily maintained. The only disadvantage is their relatively short flight endurance due to low energy efficiency. Opportunities for improvement of their aerodynamic and thrust efficiency are almost run out. It is expected that the flight endurance will be increased by developing batteries with higher specific energy $\bar{E}=\frac{E}{m_{\text {bat }}}$.

This paper presents a simple and efficient algorithm for development of copters that fulfil customer requirements.

\section{Initial Requirements}

Customers most frequency requires copters with long endurance at the payload used, which are relatively cheap, that possess high level of reliability and simple maintenance [5-7].

\section{Mathematical model}

\section{Basic properties and features}

Copter mass can be presented as the sum of the masses of individual subsystems:

$$
m_{o}=m_{\text {con }}+m_{\text {prop }}+m_{a}+m_{p}+m_{\text {bat }}[g] .
$$

Required thrust for motionless hovering is a multiplication of required thrust of one propulsor by the number of propulsor: 


$$
F_{r}=F_{1} n[N] \text {. }
$$

Required thrust of one propulsor is calculated according to the formula by accepting that $4 \%$ of the thrust is used to limit displacements caused by air movement:

$$
F_{1}=1.04 \frac{m_{o} g}{n}[N] \text {. }
$$

Total required power is:

$$
P_{r}=n f\left(F_{1}\right)[W] \text {. }
$$

Required power of one motor for motionless hovering depends on required thrust. This dependence is presented by manufacturers of electric motors for copters in tables with experimental data. In tables, for a specific motor, propeller and battery voltage, depending on revolutions, are presented data for power consumption and achieved thrust. dependence:

According to data by using a parabolic regression we can find the

$$
\begin{array}{ll}
\text { (5) } & P_{1}=f\left(F_{1}\right)[W], \\
\text { (6) } & E=P_{r} t=\bar{E} m_{\text {bat }}[\mathrm{Wh}], \\
\text { (7) } & t=\frac{E}{60 n P_{1}}=\frac{\bar{E} m_{\text {bat }}}{60 n P_{1}}[\mathrm{~min}] .
\end{array}
$$

For the electric motor U8lite KV150, with the propeller G28*9.2CF and batteries with a voltage of $24 \mathrm{~V}$, the following dependence between power consumption and required thrust can be found:

Table 1

\begin{tabular}{|c|c|}
\hline F1, $\mathbf{N}$ & P1, W \\
\hline 11.54 & 69.6 \\
\hline 12.13 & 74.4 \\
\hline 13.27 & 84.0 \\
\hline 14.37 & 93.6 \\
\hline 15.31 & 103.2 \\
\hline 16.17 & 110.4 \\
\hline 17.51 & 124.8 \\
\hline 18.69 & 134.4 \\
\hline 19.68 & 146.4 \\
\hline 21.12 & 160.8 \\
\hline
\end{tabular}




\begin{tabular}{|c|c|}
\hline F1, N & P1, W \\
\hline 21.85 & 168.0 \\
\hline 22.90 & 180.0 \\
\hline 24.03 & 194.4 \\
\hline 25.01 & 206.4 \\
\hline 26.74 & 230.4 \\
\hline 28.22 & 247.2 \\
\hline 30.89 & 283.2 \\
\hline 34.13 & 326.4 \\
\hline 39.81 & 415.2 \\
\hline 47.86 & 552.0 \\
\hline
\end{tabular}

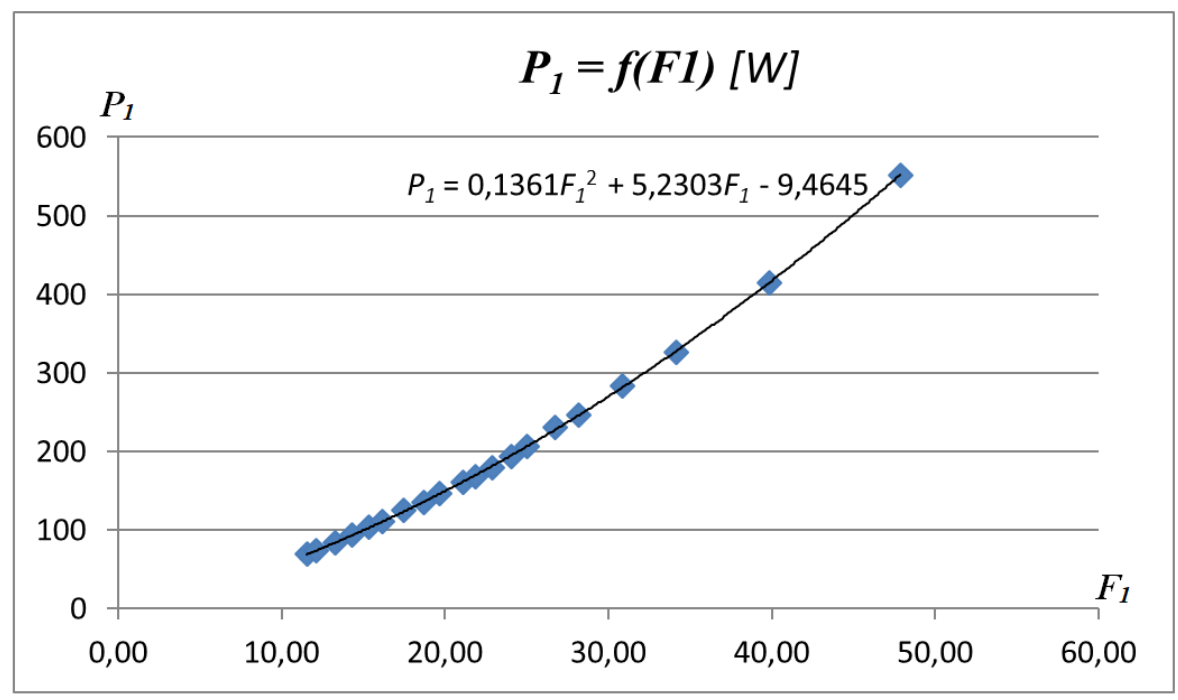

Fig. 1. Parabolic regression

For dependence between power consumption and required thrust of the electric motor U8lite KV150, with the propeller G28*9,2CF and batteries providing $24 \mathrm{~V}$.

When looking at a specific example about designing of an industrial copter, some dependence can be found, which can be used for development of a copter fulfilling customer requirements.

An example is considered where a customer requires a copter capable to carry a payload with gimbal mass of $m_{p} \leq 1500 \mathrm{~g}$ and have a flight time of 
$t \geq 90 \mathrm{~min}$. Calculations have been performed for two basic copter configurations with four rotors (quadcopters) and six rotors (sixcopters), which are the most frequency used for these payloads. It should be noted that this copter are able to carry higher loads at the expense of a reduced flight time.

After analysis of the masses of the structure, we accept:

- $m_{\text {con } 4}=1350 \mathrm{~g}$ for quadcopter;

- $m_{\text {con } 6}=2700 \mathrm{~g}$ for sixcopter;

- $m_{a}=500 \mathrm{~g}$ for avionics.

An option with a highly effective propulsion system has been considered. The system consists of brushless electric motors U8lite KV150, a propeller G28*9,2CF and batteries with a voltage of $24 \mathrm{~V}$. Flight times with different types of batteries and specific electric energy have been compared: LiPo with $\bar{E}=200 \mathrm{Wh} / \mathrm{kg}$, LiION with $\bar{E}=250 \mathrm{Wh} / \mathrm{kg}$ and promising batteries which are expected to be used in 2026 with $\bar{E}=1200 \mathrm{Wh} / \mathrm{kg}$.

Table 2

\begin{tabular}{|c|c|c|c|c|c|c|}
\hline \multirow{3}{*}{$m_{b a t}$} & \multicolumn{6}{|c|}{ Quadcopter } \\
\hline & \multirow{2}{*}{$m_{0}$} & \multirow{2}{*}{$F_{1}$} & \multirow{2}{*}{$\boldsymbol{P}$} & \multicolumn{3}{|c|}{$\overline{\boldsymbol{E}}$} \\
\hline & & & & 200 & 250 & 1200 \\
\hline $\mathbf{g}$ & g & $\mathbf{N}$ & $\mathbf{W}$ & \multicolumn{3}{|c|}{$t, \min$} \\
\hline 1500 & 6277 & 16 & 437 & 41.22 & 51.53 & 247.33 \\
\hline 1750 & 6527 & 17 & 461 & 45.52 & 56.90 & 273.12 \\
\hline 2000 & 6777 & 17 & 486 & 49.34 & 61.67 & 296.02 \\
\hline 2250 & 7027 & 18 & 512 & 52.73 & 65.92 & 316.40 \\
\hline 2500 & 7277 & 19 & 538 & 55.76 & 69.70 & 334.56 \\
\hline 2750 & 7527 & 19 & 564 & 58.46 & 73.08 & 350.77 \\
\hline 3000 & 7777 & 20 & 591 & 60.88 & 76.10 & 365.26 \\
\hline 3250 & 8027 & 20 & 619 & 63.04 & 78.79 & 378.22 \\
\hline 3500 & 8277 & 21 & 646 & 64.97 & 81.21 & 389.81 \\
\hline 3750 & 8527 & 22 & 675 & 66.70 & 83.37 & 400.18 \\
\hline 4000 & 8777 & 22 & 703 & 68.24 & 85.31 & 409.47 \\
\hline 4250 & 9027 & 23 & 732 & 69.63 & 87.04 & 417.77 \\
\hline 4500 & 9277 & 24 & 762 & 70.87 & 88.58 & 425.19 \\
\hline 4750 & 9527 & 24 & 792 & 71.97 & 89.96 & 431.82 \\
\hline 5000 & 9777 & 25 & 822 & 72.95 & 91.19 & 437.73 \\
\hline
\end{tabular}




\begin{tabular}{|r|c|c|c|r|r|r|}
\hline \multirow{2}{*}{$\boldsymbol{m}_{\text {bat }}$} & \multicolumn{6}{|c|}{ Quadcopter } \\
\cline { 2 - 7 } & \multirow{2}{*}{$\boldsymbol{m}_{\mathbf{0}}$} & $\boldsymbol{F}_{\mathbf{1}}$ & $\boldsymbol{P}$ & \multicolumn{3}{|c|}{$\overline{\boldsymbol{E}}$} \\
\hline & & & & $\mathbf{2 0 0}$ & $\mathbf{2 5 0}$ & $\mathbf{1 2 0 0}$ \\
\hline $\mathbf{g}$ & $\mathbf{g}$ & $\mathbf{N}$ & $\mathbf{W}$ & \multicolumn{3}{|c|}{$\boldsymbol{t}$ min } \\
\hline 5250 & 10027 & 26 & 853 & 73.83 & 92.29 & 442.99 \\
\hline 5500 & 10277 & 26 & 885 & 74.61 & 93.26 & 447.65 \\
\hline 5750 & 10527 & 27 & 916 & 75.30 & 94.12 & 451.78 \\
\hline 6000 & 10777 & 27 & 949 & 75.90 & 94.88 & 455.42 \\
\hline 6250 & 11027 & 28 & 981 & 76.43 & 95.54 & 458.61 \\
\hline 6500 & 11277 & 29 & 1014 & 76.90 & 96.12 & 461.39 \\
\hline 6750 & 11527 & 29 & 1048 & 77.30 & 96.63 & 463.81 \\
\hline 7000 & 11777 & 30 & 1082 & 77.65 & 97.06 & 465.88 \\
\hline 7250 & 12027 & 31 & 1116 & 77.94 & 97.43 & 467.64 \\
\hline 7500 & 12277 & 31 & 1151 & 78.19 & 97.73 & 469.12 \\
\hline 7750 & 12527 & 32 & 1186 & 78.39 & 97.99 & 470.33 \\
\hline 8000 & 12777 & 33 & 1222 & 78.55 & 98.19 & 471.30 \\
\hline 8250 & 13027 & 33 & 1258 & 78.68 & 98.34 & 472.05 \\
\hline 8500 & 13277 & 34 & 1295 & 78.77 & 98.46 & 472.60 \\
\hline 8750 & 13527 & 35 & 1332 & 78.83 & 98.53 & 472.96 \\
\hline 9000 & 13777 & 35 & 1370 & 78.86 & 98.57 & 473.15 \\
\hline 9250 & 14027 & 36 & 1408 & 78.86 & 98.58 & 473.17 \\
\hline 9500 & 14277 & 36 & 1446 & 78.84 & 98.55 & 473.06 \\
\hline 9750 & 14527 & 37 & 1485 & 78.80 & 98.50 & 472.80 \\
\hline 10000 & 14777 & 38 & 1524 & 78.74 & 98.42 & 472.43 \\
\hline 10250 & 15027 & 38 & 1564 & 78.66 & 98.32 & 471.93 \\
\hline 10500 & 15277 & 39 & 1604 & 78.56 & 98.20 & 471.34 \\
\hline 10750 & 15527 & 40 & 1645 & 78.44 & 98.05 & 470.64 \\
\hline & & & & & & \\
\hline
\end{tabular}




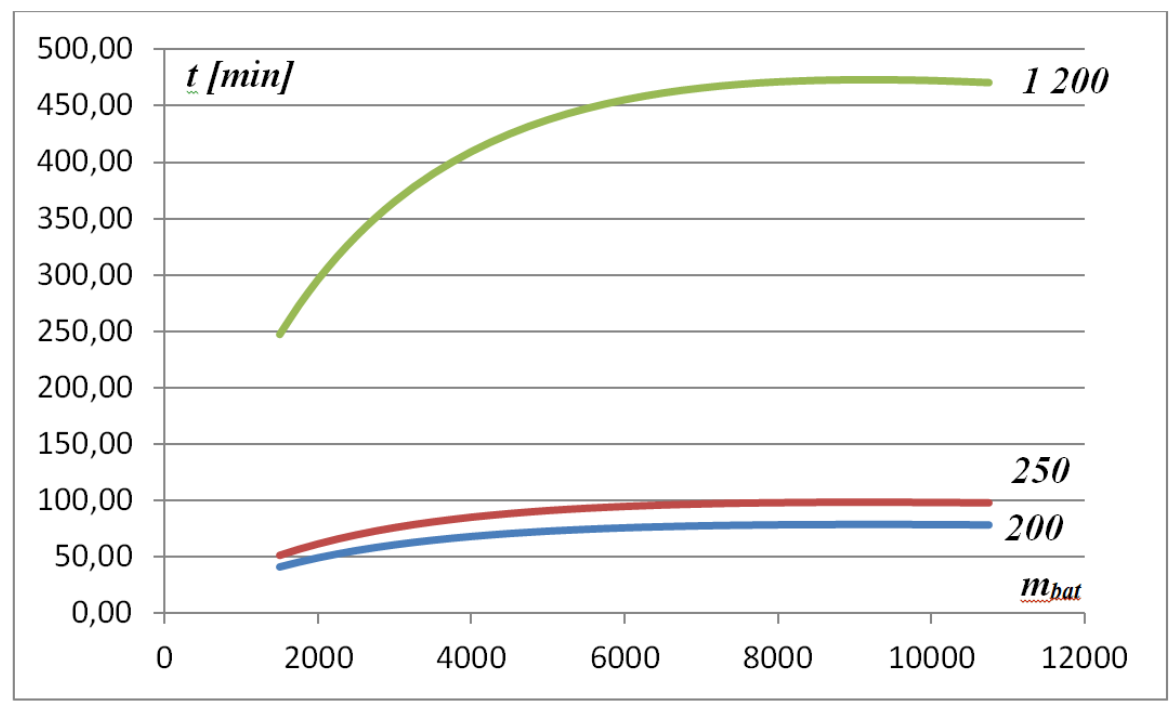

Fig. 2. Flight endurance of a quadcopter according to the mass of batteries at $\bar{E}=200,250$ and $1200 \mathrm{Wh} / \mathrm{kg}$

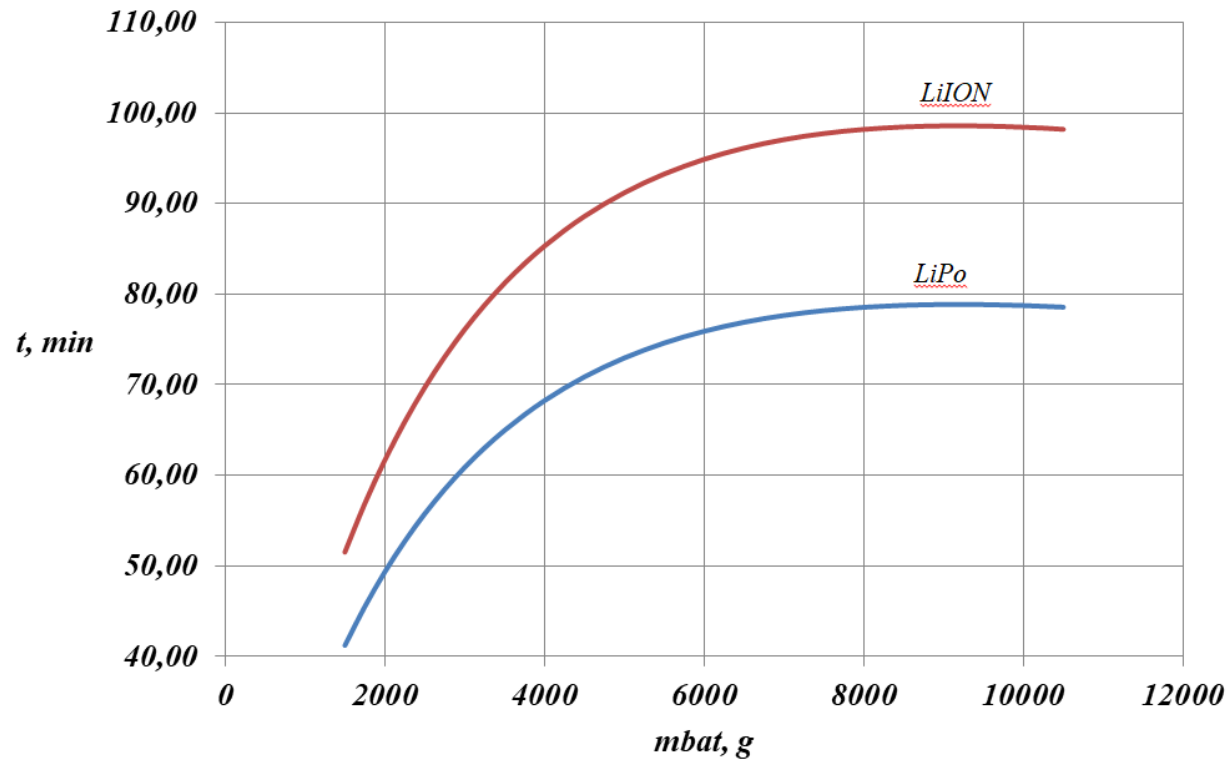

Fig. 3. Flight endurance of a quadcopter

with a battery LiPo at $\bar{E}=200$ and LiION at $\bar{E}=250 \mathrm{Wh} / \mathrm{kg}$

according to the mass of batteries 
Following conclusions can be drawn according to table data and figures:

- The maximum endurance with LiPo batteries is less than $80 \mathrm{~min}$ which do not fulfill customer requirements;

- Required flight time can be only achieved when using LiION batteries with a mass of $m_{\text {bat }}=5000 \mathrm{~g}$ and $m_{0}=9777 \mathrm{~g}$. By using these types of batteries with a higher mass, the flight endurance increases slightly. Maximum endurance of 98,58 min is reached by using batteries with a mass of $m_{b a t}=9850 \mathrm{~g}$ and $m_{0}=14027 \mathrm{~g}$;

- With promising batteries, the required endurance will be achieved at $m_{\text {bat }} \leq 1500 \mathrm{~g}$ and $m_{0} \leq 6000 \mathrm{~g}$. Promising batteries will provide flight endurance over 8.5 hours so that the usage of other sources of energy will be inefficient.

When designing, several copter configurations should be explored in order to achieve the best solution. In this case, the scheme of a sixcopter is considered.

Table 3

\begin{tabular}{|c|c|c|c|c|c|c|}
\hline \multirow{2}{*}{ mbat } & \multicolumn{6}{|c|}{ Sixcopter } \\
\cline { 2 - 7 } & \multirow{2}{*}{$\mathbf{m 0}$} & \multirow{2}{*}{$\mathbf{F 1}$} & $\mathbf{P}$ & \multicolumn{3}{|c|}{ Espec } \\
\cline { 5 - 7 } & & & & $\mathbf{2 0 0}$ & $\mathbf{2 5 0}$ & $\mathbf{1 2 0 0}$ \\
\hline $\mathbf{g}$ & $\mathbf{g}$ & $\mathbf{N}$ & $\mathbf{W}$ & \multicolumn{3}{|c|}{ t, min } \\
\hline 1500 & 8341 & 21 & 654 & 27.54 & 34.42 & 165.24 \\
\hline 1750 & 8591 & 22 & 682 & 30.79 & 38.49 & 184.77 \\
\hline 2000 & 8841 & 23 & 711 & 33.77 & 42.21 & 202.61 \\
\hline 2250 & 9091 & 23 & 740 & 36.49 & 45.61 & 218.94 \\
\hline 2500 & 9341 & 24 & 770 & 38.98 & 48.73 & 233.89 \\
\hline 2750 & 9591 & 24 & 800 & 41.27 & 51.58 & 247.59 \\
\hline 3000 & 9841 & 25 & 830 & 43.36 & 54.20 & 260.17 \\
\hline 3250 & 10091 & 26 & 861 & 45.28 & 56.61 & 271.71 \\
\hline 3500 & 10341 & 26 & 893 & 47.05 & 58.81 & 282.30 \\
\hline 3750 & 10591 & 27 & 925 & 48.67 & 60.84 & 292.04 \\
\hline 4000 & 10841 & 28 & 957 & 50.16 & 62.71 & 300.99 \\
\hline 4250 & 11091 & 28 & 990 & 51.54 & 64.42 & 309.21 \\
\hline 4500 & 11341 & 29 & 1023 & 52.80 & 65.99 & 316.78 \\
\hline 4750 & 11591 & 30 & 1056 & 53.95 & 67.44 & 323.73 \\
\hline 5000 & 11841 & 30 & 1091 & 55.02 & 68.77 & 330.11 \\
\hline 5250 & 12091 & 31 & 1125 & 56.00 & 70.00 & 335.98 \\
\hline
\end{tabular}




\begin{tabular}{|c|c|c|c|c|c|c|}
\hline \multirow{2}{*}{ mbat } & \multicolumn{6}{|c|}{ Sixcopter } \\
\cline { 2 - 7 } & \multirow{2}{*}{$\mathbf{f 0}$} & \multirow{2}{*}{$\mathbf{2}$} & $\mathbf{P}$ & \multicolumn{3}{|c|}{ Espec } \\
\cline { 5 - 8 } $\mathbf{g}$ & $\mathbf{g}$ & $\mathbf{N}$ & $\mathbf{W}$ & \multicolumn{3}{|c|}{ t, min } \\
\hline 5500 & 12341 & 31 & 1160 & 56.89 & 71.12 & 341.37 \\
\hline 5750 & 12591 & 32 & 1195 & 57.72 & 72.15 & 346.31 \\
\hline 6000 & 12841 & 33 & 1231 & 58.47 & 73.09 & 350.84 \\
\hline 6250 & 13091 & 33 & 1268 & 59.17 & 73.96 & 355.00 \\
\hline 6500 & 13341 & 34 & 1304 & 59.80 & 74.75 & 358.80 \\
\hline 6750 & 13591 & 35 & 1342 & 60.38 & 75.47 & 362.27 \\
\hline 7000 & 13841 & 35 & 1379 & 60.91 & 76.13 & 365.44 \\
\hline 7250 & 14091 & 36 & 1417 & 61.39 & 76.73 & 368.32 \\
\hline 7500 & 14341 & 37 & 1456 & 61.82 & 77.28 & 370.94 \\
\hline 7750 & 14591 & 37 & 1495 & 62.22 & 77.77 & 373.32 \\
\hline 8000 & 14841 & 38 & 1534 & 62.58 & 78.22 & 375.46 \\
\hline 8250 & 15091 & 38 & 1574 & 62.90 & 78.62 & 377.39 \\
\hline 8500 & 15341 & 39 & 1614 & 63.19 & 78.98 & 379.13 \\
\hline 8750 & 15591 & 40 & 1655 & 63.45 & 79.31 & 380.67 \\
\hline 9000 & 15841 & 40 & 1696 & 63.67 & 79.59 & 382.04 \\
\hline 9250 & 16091 & 41 & 1738 & 63.88 & 79.84 & 383.25 \\
\hline 9500 & 16341 & 42 & 1780 & 64.05 & 80.06 & 384.31 \\
\hline 9750 & 16591 & 42 & 1822 & 64.20 & 80.26 & 385.23 \\
\hline 10000 & 16841 & 43 & 1865 & 64.33 & 80.42 & 386.01 \\
\hline 10250 & 17091 & 44 & 1909 & 64.44 & 80.56 & 386.67 \\
\hline 10500 & 17341 & 44 & 1952 & 64.53 & 80.67 & 387.20 \\
\hline 10750 & 17591 & 45 & 1997 & 64.61 & 80.76 & 387.64 \\
\hline
\end{tabular}




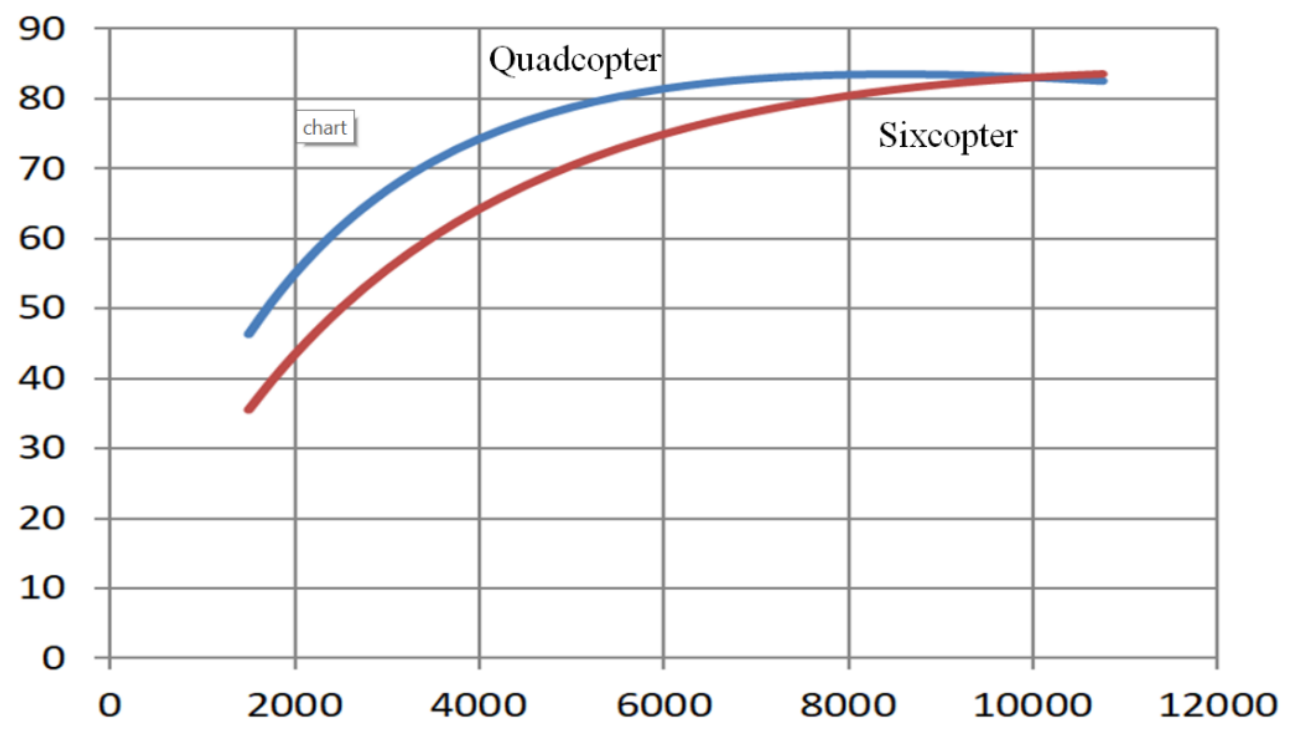

Fig. 4. Flight endurance of a quadcopter and sixcopter according to the mass of batteries at $\bar{E}=200 \mathrm{Wh} / \mathrm{kg}$

The tables and diagrams show that a sixcopter is not able to provide the required flight endurance.

\section{Conclusion}

The algorithm allows to find options that fulfil customer requirements and to eliminate options which do not fulfil these requirements and also are not competitive.

\section{Acknowledgements}

Set up under the project 9иф-02-4/28.11.2018 "Development of an innovative drone with long endurance and high altitude", financed by the National Innovation Fund of Ministry of Economic of Republic of Bulgaria.

\section{References}

1. U8 Lite KV150. T-Motor. http://store-en.tmotor.com/goods.php?id=469

2. Stojkov, O., I. Kamburov. Matematicheski model na eksploatacijata na sistemite za upravlenie na aviacionnoto v"or"zhenie. 1983 Nauchni trudove "VNVVU G. Benkovski", No. 44, VI, Sofia. (in Bulgarian) 
3. Stojkov, O. Kompleksna obrabotka na informacijata poluchavana ot razlichni datchici. 1988 Nauchni trudove "VNVVU G. Benkovski", No. 51, VI, Sofia. (in Bulgarian)

4. Stojkov, O. Optikoelektronni sistemi. NVU "V. Levski”, fakultet “Aviacionen”, Jubilejna nauchna sesija. 2005, Vol. 1, Dolna MitropoliJa, 164-170, ISBN 954-713-071-4. (in Bulgarian)

\section{МНОГОРОТОРЕН БЕЗПИЛОТЕН ЛЕТАТЕЛЕН АПАРАТ С ГОЛЯМА ПРОДЪЛЖИТЕЛНОСТ НА ПОЛЕТА}

\section{Д. Зафиров}

\section{Резюме}

В тази статия се предлага алгоритъм за проектиране на многороторен безпилотен летателен апарат с голяма продължителност на полета. Направени са пресмятания за използването на различни видове батерии, като са получени зависимости на полетното време за различни техни маси. Разгледани са варианти за четирироторен и шестроторен безпилотни летателни апарати. 